\title{
Tratamiento de la gonalgia por gonartrosis con ozono intrarticular
}

\author{
D. Samper Bernal ${ }^{1,2,3}$, G. Rovira Dupláa ${ }^{3}$, M. M. Monerris Tabasco ${ }^{2}$, M. González Palomares ${ }^{3}$ \\ y V. Mazo Sánchez ${ }^{2}$ \\ ${ }^{1}$ Unidad del Dolor. ${ }^{2}$ Servicio de Anestesiología y Reanimación. ${ }^{3}$ Institut Català d'Ozonoteràpia. \\ Hospital Universitari Germans Trias i Pujol.
}

Samper Bernal D, Rovira Dupláa G, Monerris Tabasco MM, González Palomares M, Mazo Sánchez V. Tratamiento de la gonalgia por gonartrosis con ozono intrarticular. Rev Soc Esp Dolor 2013; 20(3): 107-112.

\begin{abstract}
Objective: To determine the efficacy of intra-articular knee infiltration with medical ozone in patients with chronic knee pain due to knee osteoarthritis.

Methods: An observational, prospective and intrasubject, included 203 patients with knee osteoarthritis and pain secondary to most, there were a total of five intra-articular injections with medical ozone at a concentration of $25 \mathrm{ug} / \mathrm{ml}$ and a volume of $10 \mathrm{ml}$ at weekly intervals. Analgesic quality was evaluated by visual analogue scale (VAS) and pain (WOMAC-A), stiffness (WOMAC-B) and functional capacity (WOMAC-C) using the questionnaire Western Ontario and McMaster Universities Osteoarthritis (WOMAC) before and after treatment.

Results: 203 patients were performed a total of 305 procedures, 204 women and 101 men, with a mean age of $69.9 \pm$ 9.4 years. Giving to EVA, WOMAC-A,-B and WOMAC WOMAC-C range of quantitative variables, the mean pre-treatment were $6.88 \pm 2.30,8.12 \pm 3.76,3.39 \pm 1.88$ and $29.15 \pm$ 13.04, respectively, and the mean response after treatment, $3.98 \pm 2.35,4.61 \pm 2.79,2.10 \pm 1.50$ and $17.51 \pm 10.36$ respectively. The differences between the responses before and after treatment, compared with Student's t tests for paired data were all significant at $\mathrm{p}<0.001$.

Conclusion: The use of an intra-articular ozone concentration of 25 ug / $\mathrm{ml}$ and a volume of $10 \mathrm{ml}$ is effective for the relief of chronic pain secondary to knee osteoarthritis. As most
\end{abstract}

Recibido: 31-07-12.

Aceptado: 01-11-12 frequent complication was recorded mild pain during the administration of ozone, followed by feeling of occupation and joint crepitation so we can consider it also as a safe treatment free of systemic effects have required medical interventions.

Key works: Arthritis knee. Pain. Infiltration. Ozone.

\section{RESUMEN}

Objetivo: Determinar la eficacia de la infiltración intrarticular de la rodilla con ozono médico en pacientes que presentan gonalgia por gonartrosis crónica.

Método: Estudio observacional, prospectivo e intrasujeto; se incluyeron 203 pacientes con gonalgia secundaria a gonartrosis en su mayoría; se realizaron un total de 5 infiltraciones intrarticulares con ozono médico, a una concentración de $25 \mu \mathrm{g} /$ $\mathrm{ml}$ y un volumen de $10 \mathrm{ml}$, a intervalos semanales. Se evalúo la calidad analgésica mediante la escala visual analógica (EVA) y el dolor (WOMAC-A), rigidez (WOMAC-B) y capacidad funcional (WOMAC-C) utilizando el cuestionario Western Ontario and McMaster Universities Osteoarthritis (WOMAC), antes y después del tratamiento.

Resultados: A los 203 pacientes se le realizaron un total de 305 procedimientos, 204 fueron realizados en mujeres y 101 en hombres, siendo la media de edad de 69,9 \pm 9,4 años. Otorgando a EVA, WOMAC-A, WOMAC-B y WOMAC-C rango de variables cuantitativas, las medias previas al tratamiento fueron $6,88 \pm 2,30,8,12 \pm 3,76,3,39 \pm 1,88$ y $29,15 \pm$ 13,04 respectivamente y las medias de respuesta posteriores al tratamiento, $3,98 \pm 2,35,4,61 \pm 2,79,2,10 \pm, 1,50$ y 17,51 $\pm, 10,36$ respectivamente. Las diferencias entre las respuestas previas y posteriores al tratamiento, comparadas con el test de $\mathrm{T}$ de student para datos apareados fueron todas ellas significativas con $p<0,001$.

Conclusión: El uso de ozono intrarticular a una concentración de $25 \mu \mathrm{g} / \mathrm{ml}$ y un volumen de $10 \mathrm{ml}$ es eficaz para el alivio del dolor crónico secundario a gonartrosis. Como complicación más frecuente se registró dolor leve durante la administración 
del ozono, seguida de sensación de ocupación y crepitación articular por lo que también lo podemos considerar como un tratamiento seguro exento de repercusiones sistémicas que hayan requerido de actuaciones médicas.

Palabras clave: Gonartrosis. Dolor. Infiltración. Ozono.

\section{INTRODUCCIÓN}

Una de las localizaciones más frecuentes de osteoartrosis $(\mathrm{OA})$ es en la rodilla. En España la artrosis sintomática de rodilla se presenta en el 10,2\% de las personas mayores de 20 años (1). Además hay que considerar que el riesgo de discapacidad atribuible solo a la artrosis de rodilla es equivalente a las cardiopatías y superior al de cualquier otro trastorno médico en el anciano (2).

Tradicionalmente el diagnóstico de la enfermedad es clínico y/o radiológico. La clasificación radiológica más comúnmente utilizada es la establecida por KellgrenLawrence en 1957 (3). La forma ideal para el diagnóstico de la OA debe incluir al menos criterios radiológicos y clínicos, siendo los criterios clínicos definidos por el American College of Rheumatology los más ampliamente utilizados (4).

Dada la distinta etiopatogenia de la artrosis y diferente historia natural, en el año 2008 fueron publicadas las recomendaciones de la Osteoarthritis Research Society International (OARSI) para el tratamiento de la artrosis de cadera y rodilla (5), proponiéndose 25 recomendaciones, 12 no farmacológicas, 8 farmacológicas -entre estas las infiltraciones intrarticulares con corticoides y ácido hialurónicoy 5 quirúrgicas. Los objetivos principales del tratamiento deben ser: la educación de los pacientes, la reducción y el control del dolor, el mantenimiento de la movilidad de la rodilla y la reducción de la progresión de la artrosis.

El ozono médico es una mezcla (un $95 \%$ de $\mathrm{O}_{2}$ y un 5 $\%$ de $\mathrm{O}_{3}$ ) utilizada en varias concentraciones y administrada por diferentes vías. Es un gas inestable, transparente y de olor picante. El ozono se produce a partir del oxígeno cuando entra en contacto con una descarga eléctrica que genera diferentes concentraciones según la enfermedad a tratar (6). Su acción antinflamatoria se debe a la inhibición de prostaglandinas, incrementando la liberación de antagonistas de citocinas proinflamatorias.

Activa mecanismos antioxidantes endógenos y produce una metabolización de radicales libres, frenando el proceso oxidativo responsable de la destrucción celular. El estrés oxidativo está presente en diversos procesos como la inflamación, enfermedades degenerativas, deficiencias inmunitarias, enfermedades autoinmunes, etc. Su correcta utilización puede mejorar la función celular, aumentando la capacidad de frenar los procesos fisiológicos y patológicos de destrucción celular (7).
La acción antioxidante a nivel intrarticular disminuye la inflamación y la futura degeneración. Inactiva e inhibe la liberación de enzimas proteolíticas. Estimula la producción de condrocitos y fibroblastos con la posibilidad de formación de nuevo cartílago.

Tiene un efecto antinflamatorio y analgésico. Esta acción es consecuencia de una inhibición de la síntesis de prostaglandinas (8) o la liberación de bradicinina y otros mediadores de la inflamación. Aumenta la liberación de antagonistas que neutralizan proinflamatorios como citoquinas like (interleuquinas, interferón, factor $\alpha$ de necrosis tumoral y citoquinas inmunosupresoras like), todos con efecto inhibidor de la inflamación. Libera endorfinas, que bloquea la transmisión de la señal nociceptiva hacia el tálamo y la corteza. Todo en conjunto produce una disminución del edema, la inflamación y el dolor $(9,10)$.

Actualmente la administración de ozono intrarticular en la artrosis de rodilla no está recomendada en las guías de práctica clínica, sin embargo su administración intrarticular tiene como objetivo fundamental tratar los síntomas a corto y medio plazo mediante la reducción mantenida del dolor, la conservación de la movilidad y la reducción de la incapacidad funcional.

El problema de la artrosis no es un problema exclusivamente mecánico, sino que existen alteraciones celulares y bioquímicas que justifican los resultados obtenidos con la aplicación de ozono de pequeñas cantidades peri e intrarticulares (11). Su aplicación no tiene efectos secundarios y se ha demostrado útil en cuanto a la mejoría del dolor, la inflamación y consecuentemente de la función $(12,13)$.

El ozono no tiene ningún riesgo a corto o medio plazo. La desventaja principal es su lento inicio de acción, que hace que en algunos casos no se consiga una reducción del dolor hasta pasadas unas semanas. Únicamente en los pacientes con favismo (déficit de glucosa-6-fosfato deshidrogenasa) está contraindicada su administración.

Son escasos los estudios publicados de la administración de ozono en la artrosis de rodilla. Fahmy (14) y RivaSanseverino (15) son los primeros autores que abogan por su uso en la artrosis de rodilla y la artritis reumatoide como tratamiento de soporte. En 1998, Escarpenter (16) reporta 126 pacientes tratados con ozono. También, Riva Sansaverino (17) en otro estudio de 83 pacientes con artrosis de rodilla, informa de una mejoría del dolor articular.

Milanés (18) obtuvo un 88,1\% de resultados satisfactorios respecto al EVA y la función articular -mediante el uso de un goniómetro para medir la amplitud del arco articular- en un grupo de 321 pacientes.

Recientemente, Cabot (19) ha presentado un estudio en el que incluye a 57 pacientes (73 rodillas) afectos de gonartrosis tricompartimental o de condropatía rotuliana, a los que se les aplicó de manera combinada ozonoterapia y factores de crecimiento de origen plaquetario, 
obteniendo resultados satisfactorios en el 82 y $87 \%$ respectivamente.

\section{MATERIAL Y MÉTODOS}

Se realizó un estudio observacional, prospectivo e intrasujeto, en el que se incluyeron un total de 203 pacientes afectos de gonalgia secundaria a gonartrosis en los que había fracasado el tratamiento conservador previo. Dicho estudio se llevó a cabo durante un periodo de 12 meses. Se obtuvo el consentimiento informado por escrito por parte del paciente.

La patología de origen era la gonartrosis. Todos los pacientes realizaban o habían realizado tratamiento médico y/o fisioterápico asociado bien conducido, habiéndoles indicado en la mayoría de ellos cirugía protésica articular. Antes de la administración intrarticular del ozono médico se recogieron de los pacientes datos demográficos, intensidad del dolor (valorada mediante escala visual analógica -EVA-) y puntuación en el cuestionario WOMAC en sus respectivos apartados.

La vía de abordaje en la articulación de la rodilla fue la pararrotuliana (anteroexterno o anterointerno) entrando en el fondo de saco de la rodilla; el paciente se encontraba sentado con la pierna flexionada $90^{\circ}$.

Se usó lidocaína $1 \%$ para anestesiar la piel y tejido subcutáneo de la zona de punción. Se utilizó un generador de ozono médico. Se utilizó aguja tipo Quincke de 27G de $4 \mathrm{~cm}$. La dosis de ozono intrarticular fue de $10 \mathrm{ml}$ en cada rodilla afecta, a una concentración de $25 \mu \mathrm{g} / \mathrm{ml}$. Se realizó en cada rodilla afecta un total de 5 sesiones, en intervalos de 7 días entre ellas.

Posteriormente a las 5 sesiones de ozonoterapia intrarticular se realizó un control médico, recogiéndose los efectos adversos, y los cambios producidos en la intensidad del dolor mediante la aplicación de la EVA y la puntuación en el cuestionario WOMAC en sus diferentes apartados (WOMAC-A: analgesia, WOMAC-B: rigidez articular y WOMAC-C: capacidad funcional). De esta manera se ha evaluado el cambio en la respuesta como consecuencia de un tratamiento, en nuestro caso con la administración intrarticular de ozono médico.

Se compararon las medias de EVA, WOMAC-A, WOMAC$\mathrm{B}$, WOMAC-C entre antes y después del tratamiento.

Se calculó el cambio porcentual de los valores de EVA, WOMAC-A, WOMAC-B y WOMAC-C (estimados como variables cuantitativas) entre los tomados antes y después del tratamiento y se compararon resultados entre hombres y mujeres.

Los cambios porcentuales fueron categorizados en diez tramos iguales entre 0 y $100 \%$ con el fin de calcular qué porcentaje de procedimientos se beneficiaban de descensos en los diferentes cuestionarios evaluados para cada una de las categorías.
Se compararon medias de edad y peso entre cada uno de los tramos y la proporción de hombres y mujeres dentro de cada uno de los mismos.

Las comparaciones cuantitativas entre valores pre y postratamiento intrasujetos se realizaron con test de $\mathrm{T}$ de Student para datos apareados, las comparaciones cuantitativas entre sexos con test de $\mathrm{T}$ para datos independientes. Las diferencias de edad y peso entre los diferentes tramos se analizaron con test de ANOVA y la comparación de proporción de hombres y mujeres en los diferentes tramos de las variables categorizadas se realizó con test de $\mathrm{c}^{2}$.

Se procesaron los datos con el paquete estadístico SPSS $^{\odot}$ (IBM SPSS Statistics 15, Chicago, IL). Se tomó como estadísticamente significativa una $\mathrm{p}<0,05$.

\section{RESULTADOS}

En nuestra muestra de 203 pacientes, encontramos una mayoría de mujeres $(66,9 \%)$ con una edad media de 69,93 años, con una desviación estándar (S) de 9,4 años. La media de peso fue de $76,22 \mathrm{~kg}$, con una $\mathrm{S}$ de $13,52 \mathrm{~kg}$. Se realizaron un total de 305 procedimientos (102 bilaterales y 101 unilaterales).

Las medias de EVA, WOMAC-A, WOMAC-B y WOMAC-C previas al tratamiento fueron 6,88 $\pm 2,30,8,12$ $\pm 3,76,3,39 \pm 1,88$ y $29,15 \pm 13,04$ respectivamente y las medias posteriores al tratamiento, $3,98 \pm 2,35,4,61 \pm 2,79$, $2,10 \pm 1,50$ y $17,51 \pm 10,36$ respectivamente. Las diferencias entre las respuestas previas y posteriores al tratamiento fueron todas ellas significativas con $p<0,001$.

Las disminuciones porcentuales en los valores de los diferentes cuestionarios después del tratamiento fueron: $41,95 \%$ en la escala EVA, 35,68 \% en WOMAC-A, 29,89 $\%$ en WOMAC-B y $32,13 \%$ en WOMAC-C. Las diferencias entre hombres y mujeres en la disminución de esos porcentajes no fueron significativas (Tabla I).

Dentro de cada uno de los diez tramos de porcentaje de descenso analizados para cada una de las escalas y cuestionarios estudiados, el test de ANOVA para comparar medias de edad y peso no mostró diferencias significativas y tampoco hubo diferencias significativas en la proporción de hombres y mujeres para los diferentes valores de esos tramos (Tabla II).

Las figs. 1-4 muestran el porcentaje de procedimientos que hubo en cada uno de los tramos de porcentaje de descenso postratamiento analizados en las variables EVA, WOMAC-A, WOMAC-B y WOMAC-C respectivamente.

Como única complicación podemos destacar la presencia de dolor leve durante la administración del ozono médico en un $10 \%$ de los pacientes, en forma de ocupación y crepitación articular, de unos minutos de duración.

No se registró ninguna repercusión sistémica que requiriera actuación médica. 
TABLA I. VARIACIÓN DE EVA Y WOMAC ENTRE SEXOS (DESPUÉS DEL TRATAMIENTO)

\begin{tabular}{llllll}
\hline & Sexo & $N$ & Media & $\begin{array}{c}\text { Desviación } \\
\text { típica }\end{array}$ & $\begin{array}{c}\text { Error típico } \\
\text { de la media }\end{array}$ \\
\hline \multirow{2}{*}{ Porcentaje de disminución del EVA } & Hombre & 89 & 42,2294 & 29,43321 & 3,11991 \\
& Mujer & 185 & 41,8160 & 32,28523 & 2,37366 \\
\hline \multirow{2}{*}{ Porcentaje de disminución del WOMAC (A) } & Hombre & 101 & 30,9902 & 60,15365 & 5,98551 \\
& Mujer & 204 & 38,0011 & 48,16795 & 3,37243 \\
\hline \multirow{2}{*}{ Porcentaje de disminución del WOMAC (C) } & Hombre & 101 & 27,2500 & 56,79448 & 5,65126 \\
& Mujer & 204 & 34,5471 & 57,25234 & 4,00847 \\
\hline \multirow{2}{*}{ Porcentaje de disminución del WOMAC (B) } & Hombre & 92 & 23,6465 & 66,18583 & 6,90035 \\
\end{tabular}

\section{DISCUSIÓN}

Respecto a la eficacia del tratamiento de la gonalgia por gonartrosis con ozono médico, solo cabe mostrar los datos de una manera descriptiva y someterlo a juicio clínico de los porcentajes de pacientes hallados para cada escalón de mejora.

Evidentemente, las diferencias de EVA, WOMAC (A, B y C) antes y después del procedimiento pueden ser muy significativas si se considera relevante por ejemplo un descenso de 1 punto en el EVA. Sin embargo, si lo que se considera clínicamente relevante son descensos del orden del $50 \%$ para las diferentes variables de medida utilizadas, entonces una muestra (la adecuada a esa exigencia) mucho menor a la presentada, nos hubiese mostrado significaciones mucho menores al practicar los mismos tests estadísticos.

TABLA II. VARIACIÓN DE EVA Y WOMAC PARA EDAD Y PESO (DESPUÉS DEL TRATAMIENTO)

\begin{tabular}{|c|c|c|c|c|c|c|c|}
\hline & & Edad & Peso & $\begin{array}{c}\text { Porcentaje de } \\
\text { disminución } \\
\text { del EVA }\end{array}$ & $\begin{array}{c}\text { Porcentaje } \\
\text { de dismin. } \\
\text { del WOMAC } \\
\text { (A) }\end{array}$ & $\begin{array}{c}\text { Porcentaje } \\
\text { de dismin. } \\
\text { del WOMAC } \\
\text { (B) }\end{array}$ & $\begin{array}{c}\text { Porcentaje } \\
\text { de dismin. } \\
\text { del WOMAC } \\
\text { (C) }\end{array}$ \\
\hline \multirow{2}{*}{ Edad } & Correlación de Pearson & 1 & $-0,138 *$ & $-0,058$ & $-0,028$ & $-0,061$ & $-0,002$ \\
\hline & Sig. (bilateral) & & 0,016 & 0,335 & 0,627 & 0,307 & 0,973 \\
\hline \multirow{2}{*}{ Peso } & Correlación de Pearson & $-0,138 *$ & 1 & $0,165 * *$ & 0,058 & 0,022 & $-0,002$ \\
\hline & Sig. (bilateral) & 0,016 & & 0,006 & 0,311 & 0,709 & 0,966 \\
\hline \multirow{2}{*}{$\begin{array}{l}\text { Porcentaje de } \\
\text { disminución } \\
\text { del EVA }\end{array}$} & Correlación de Pearson & $-0,058$ & $0,165 * *$ & 1 & $0,517 * *$ & $0,404 * *$ & $0,490 * *$ \\
\hline & Sig. (bilateral) & 0,335 & 0,006 & & 0,000 & 0,000 & 0,000 \\
\hline \multirow{2}{*}{$\begin{array}{l}\text { Porcentaje de } \\
\text { disminución del } \\
\text { WOMAC }(\mathrm{A})\end{array}$} & Correlación de Pearson & $-0,028$ & 0,058 & $0,517 * *$ & 1 & $0,375^{* *}$ & $0,572 * *$ \\
\hline & Sig. (bilateral) & 0,627 & 0,311 & 0,000 & & 0,000 & 0,000 \\
\hline \multirow{2}{*}{$\begin{array}{l}\text { Porcentaje de } \\
\text { disminución del } \\
\text { WOMAC (B) }\end{array}$} & Correlación de Pearson & $-0,061$ & 0,022 & $0,404 * *$ & $0,375^{* *}$ & 1 & $0,396 * *$ \\
\hline & Sig. (bilateral) & 0,307 & 0,709 & 0,000 & 0,000 & & 0,000 \\
\hline \multirow{2}{*}{$\begin{array}{l}\text { Porcentaje de } \\
\text { disminución del } \\
\text { WOMAC }(\mathrm{C})\end{array}$} & Correlación de Pearson & $-0,002$ & $-0,002$ & $0,490 * *$ & $0,572 * *$ & $0,396 * *$ & 1 \\
\hline & Sig. (bilateral) & 0,973 & 0,966 & 0,000 & 0,000 & 0,000 & \\
\hline
\end{tabular}




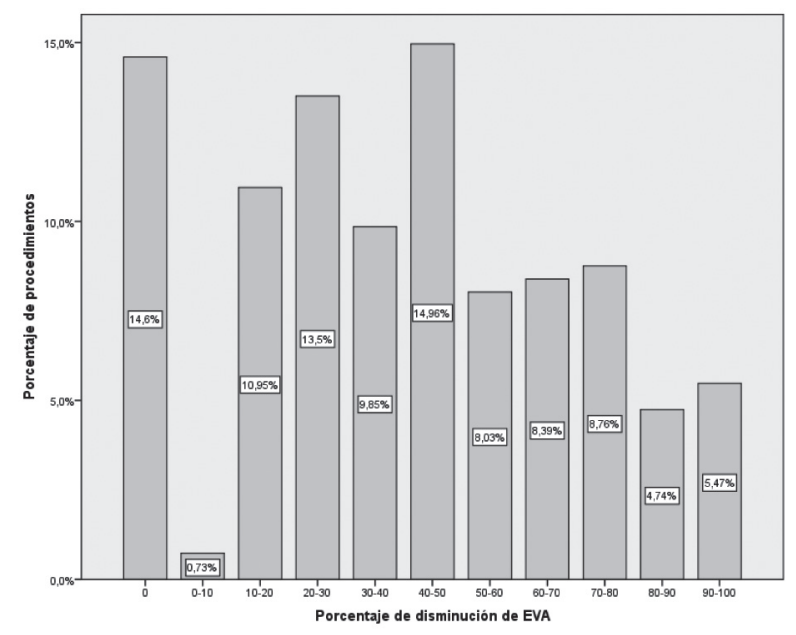

Fig. 1. Porcentaje de disminución del EVA después del tratamiento.

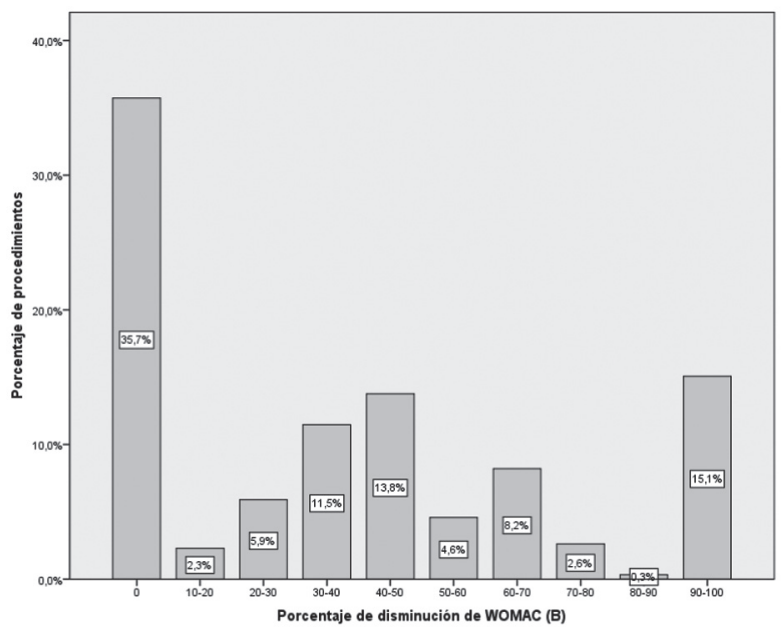

Fig. 3. Porcentaje de disminución del WOMAC-B después del tratamiento.

Es así, que en el estudio que nos ocupa, nos parece relevante el valor descriptivo de la muestra, sabiendo que si exigimos $30 \%$ de mejora para estar satisfechos con el tratamiento estaríamos en niveles de significación estadística, pero si esa exigencia se eleva al $50 \%$, con las cifras constatadas probablemente los resultados no hubiesen sido significativos. En cualquier caso, parece evidente que descensos de un $30 \%$ no pueden ser desdeñados, independientemente de que el óptimo deseable se situase en niveles superiores.

Los valores de mejoría de la analgesia evaluada con la EVA y el test de WOMAC-A, de la rigidez evaluada con el test de WOMAC-B y de la capacidad funcional evaluada con el WOMAC-C son estadísticamente significativos.

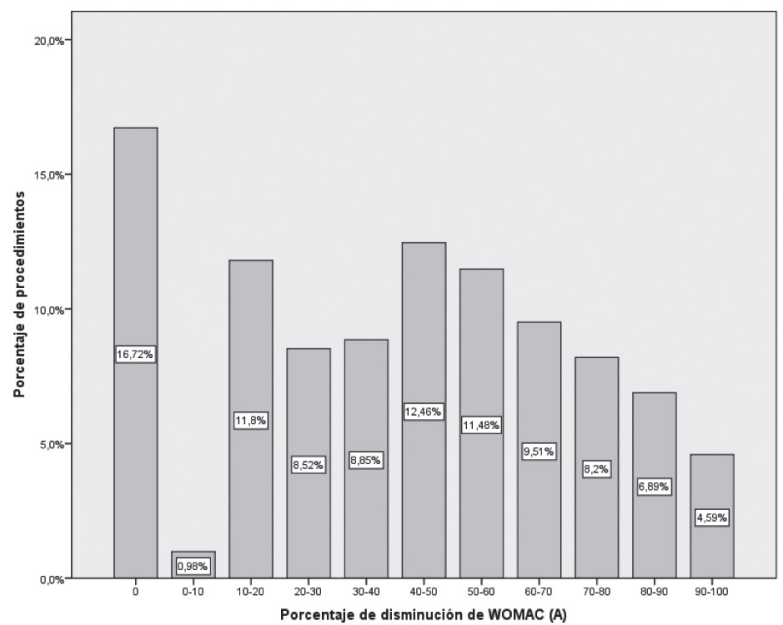

Fig. 2. Porcentaje de disminución del WOMAC-A después del tratamiento.

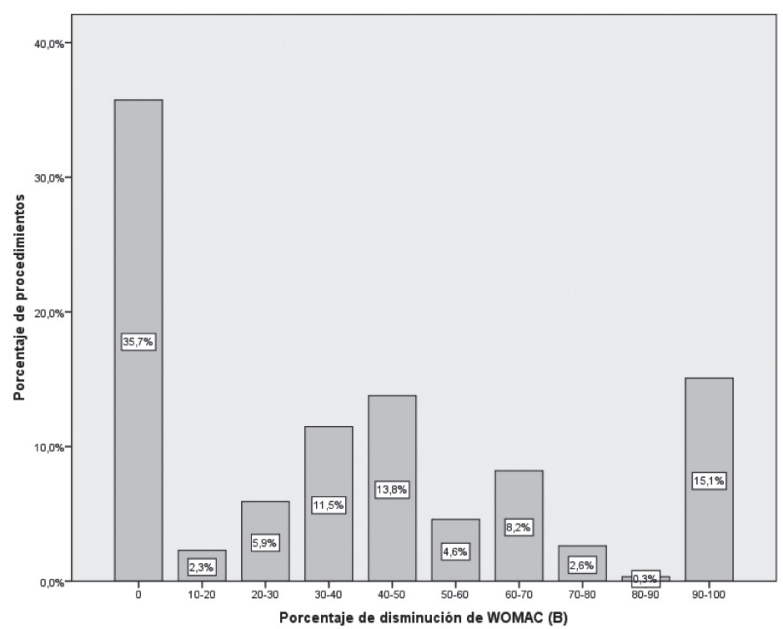

Fig. 4. Porcentaje de disminución del WOMAC-C después del tratamiento.

A la luz de los resultados, ni el peso, ni la edad ni el sexo parecieron influir en los descensos de EVA, WOMAC-A, WOMAC-B y WOMAC-C (en sus dos versiones cuantitativa y cualitativa) provocados por el tratamiento de ozono en la gonalgia.

Como ya han publicado otros autores, el uso de la mezcla de ozono se muestra útil en el tratamiento del dolor de los procesos articulares artrósicos iniciales como condropatías postraumáticas de carácter externo (accidente) o inclusive iatrogénico (artroscopias de repetición) y/o síndromes rotulianos con fases de malacia o edema de cartílago. Pero también en las artrosis evolucionadas con afectación de uno o más compartimentos si se mantiene una estabilidad articular competente, y ocasionalmente en función del terreno 
(edad, patologías asociadas, rechazo absoluto a la cirugía, etc.), podría aplicarse con una esperanza aceptable de respuesta en artrosis muy evolucionadas.

Debería poderse prever con mayor certeza la duración del periodo de alivio de las series de tratamientos. La prolongación del tratamiento con 2-3 sesiones más probablemente podría incrementar el alivio analgésico final, también queda por determinar las diferencias analgésicas potenciales entre volúmenes superiores a $10 \mathrm{ml}$ y a concentraciones inferiores a $25 \mu \mathrm{g} / \mathrm{ml}$.

Se ha de insistir en la necesidad de estudios de series largas y homogéneas, comparativos o doble ciego con valoración de escalas funcionales y un seguimiento adecuado, para poder validar de la mejor forma posible la eficacia del tratamiento con ozono. Es importante que poco a poco la medicina tradicional acepte la ozonoterapia en el tratamiento del dolor de rodilla y entre en el arsenal terapéutico habitual.

Por último insistir en algo muy importante de este tipo de tratamiento que es la falta de complicaciones relevantes, excepto el dolor leve muy transitorio, si se cumplen las reglas de aplicación y dosificación, así como las escasísimas contraindicaciones que tiene.

En resumen, el efecto terapéutico positivo del ozono médico intrarticular en gonalgias ofrece una analgesia significativa de inicio rápido y durante 3-6 meses. La mayoría de autores recomiendan repetir el ciclo terapéutico 6 meses después, ya que aproximadamente un $6 \%$ (20) pueden recidivar después de este tiempo. Su efecto terapéutico prolongado se debe a que este gas actúa de forma fisiológica, regulando entre otras acciones el metabolismo celular y la oxigenación tisular, evita los cambios degenerativos del cartílago articular, disminuye la síntesis de IL-8 y reduce la cantidad de granulocitos y de otras células inflamatorias.

\section{AGRADECIMIENTOS}

Los autores agradecen especialmente a la coordinadora M. ${ }^{a}$ Angels Durán y a la enfermera del Institut Català d'Ozonoteràpia, Raquel Montilla su inestimable colaboración durante la ejecución de este estudio. También al resto de profesionales de la Unidad.

\section{CORRESPONDENCIA:}

Institut Català d'Ozonoteràpia

Mare de Deu de la Salut, 78, 08024 Barcelona

Teléfono: 932848220

Hospital Universitari Germans Trias i Pujol

Crta. Del Canyet s/n, 08916 Badalona (Barcelona)

Teléfono: 934978929

\section{BIBLIOGRAFÍA}

1. Carmona L, Ballina J, Gabriel R, et al. EPISER Study Group. The burden of musculoskeletal diseases in the general population of Spain: results from a national survey. Ann Rheum Dis. 2001;60:1040-5.

2. Guccione AA, Felson DT, Anderson JJ, et al. The effects of specific medical conditions on the functional limitations of elders in the Framingham Study. Am J Public Health. 1994; 84(3):351-8.

3. Kellgren JH, Lawrence JS. Radiological assessment of arthrosis. Ann Rheum Dis. 1957; 16:494-502.

4. Altman R, Asch E, Bloch D, et al. Development of criteria for the classification and reporting of osteoarthritis. Classification of osteoarthritis of the knee. Diagnostic and Therapeutic Criteria Committee of the American Rheumatism Association. Arthritis Rheum. 1986;29:1039-49.

5. Zhang W, Moskowitz RW, Nuki G, et al. OARSI recommendations for the management of hip and knee osteoarthritis, Part II: OARSI evidence-based, expert consensus guidelines. Osteoarthritis Cart. 2008;16:137-62.

6. Bocci V. General mechanisms of action of ozone therapy and mechanisms in pain treatment. Rev Soc Esp Dolor. 2005;12: N. ${ }^{\circ}$ Extra. II:24-36.

7. Bocci V. Oxygen-ozono therapy. Países Bajos, Kluwer Academic Publishers, 2002.

8. Bocci V, Luzzi, Corradeschi F et al. Studies on the biological effects of ozone: III and attempt to define conditions for optimal induction of cytokines. Lymphokine Cytokine Res 1993; 12:121-6.

9. Bocci V. Mecanismos de acción generales de la ozonoterapia y mecanismos en el tratamiento del dolor. Rev Soc Esp Dolor. 2005; 12 Extra II:24-36.

10. Viebahn-Haensler R. Milestones of medical ozone. Rev Soc Esp Dolor. 2005;12 N. ${ }^{\circ}$ Extra. II:3-9.

11. de Lucas JC. Peripheral infiltrations with ozone. Indications, techniques and clinical experience. Rev Soc Esp Dolor. 2005;12 N. ${ }^{\circ}$ Extra. II:37-47.

12. Iliakis E. Utilizzo dell' ossigeno-ozonoterapia nella práctica ortopédica. Acta Toxicol Ther. 1996; XVII(2,3):249-53.

13. Gheza G, Bissolotti L. Ossigeno-Ozonoterapia mediante infiltrazione intraarticolare nella patología del ginocchio. Riv It Ossigeno-Ozonoterapia. 2003;2:63-6.

14. Fahmy Z. Ozon-sauerstofftherapie in der rheumatologie. Proceedings 5. ozon-weltkongress. Wasser Berlin. 1981.

15. Riva-Sanseverino E. Knee-joint disorders treated by oxygenozone therapy. Europa Medicophysica. 1989;25:163-70.

16. Escarpenter SC, Eden J, Suanborg A. Osteoarthritis in the elderly clinical and radiological finding in 79-85 years old. Ann Rheum Dis. 1998;12:5035-9.

17. Riva Sanseverino E. Trastornos de las articulaciones de las rodillas tratadas con oxígeno-ozono. Instituto de Fisiología Humana. Universidad de Bolonia. 2002.

18. Milanés JR, Acosta W, Trinchet E, et al. La ozonoterapia/resultados del tratamiento en pacientes con artrosis degenerativa sintomática de rodilla. Revista Mexicana de Algología. 2009; volumen 6, número IV: 18-23.

19. Cabot J. Técnica combinada de ozonoterapia y factores de crecimiento en la artrosis de rodilla. Revista Española de Ozonoterapia 2012;2. Suppl. 1:35.

20. Escarpenter SC, Valdéz O, Sánchez R, et al. Resultados terapéuticos en la osteoartritis de rodillas con infiltraciones de ozono. Rev Cubana Invest Biomed. 1997;16(2):124-32. 